\title{
VALORACIÓN DE LAS FUENTES PARA EL ANÁLISIS DEL SECTOR INDUSTRIAL EN ARAGÓN'
}

\author{
María Pilar ALONSO LOGROÑO \\ Departamento de Geografía \\ Universidad de Santiago de Compostela
}

\begin{abstract}
Resumen: El trabajo analiza la problemática de la consulta de las fuentes estadísticas para los estudios industriales. Para ello primero plantea unas reflexiones sobre las dificultades generales con los que se tiene que enfrentar el investigador en relación con el tema. Después examina las principales fuentes a las que se puede acudir para el análisis de las localizaciones industriales, tomando como referencia las existentes para la región aragonesa. En cada una plantea su nivel de acceso, sus contenidos y sus problemas.
\end{abstract}

Palabras claves: Fuentes estadísticas, localización industrial, registro industrial.

Abstract: This paper analyses the problems of using statistical sources for the study of industrial locations. First, it sets up some reflections about the general dificulties a researcher has to face relation to statistical information. After that, it goes through the main sources available to the analysis of industrial locations, specially in the aragonese region. For each one source, it studies its accesibility, contents and problems.

Key words: stadistical sources, industrial location, industrial registry.

\section{INTRODUCCIÓN}

En el camino de la investigación, la falta de experiencia y el insuficiente conocimiento de las fuentes estadísticas ocasionan muchos pasos en falso y muchas pérdidas de tiempo por no haber reflexionado a priori.

El investigador, y en especial el investigador de las ciencias sociales, no puede realizar ningún trabajo serio sin acudir a la consulta de distintas estadísticas, pero

\footnotetext{
I Mi agradecimiento a la profesora Luisa María FRUTOS, de la Universidad de Zaragroza, porque muchas de las reflexiones que contiene este árticulo provienen de sus enseñanzas.
} 
también es cierto que no sólo se deben consultar y tomar sin más las informaciones que éstas aportan sino que se debe realizar un muestreo de ellas para conocer su grado de fiabilidad. Por ello se ha creído interesante aportar nuestra experiencia en el manejo de las fuentes de información existentes para el análisis de la actividad industrial en Aragón. No se trata de plantear un trabajo puramente de contenido, ni una descripción detallada de cada una de las fuentes existentes, sino de ver las ventajas, inconvenientes, errores, vacíos ,o accesibilidad de las principales.

Junto a estos aspectos se ha querido también incorporar una serie de reflexiones generales, que no por conocidas son menos importantes, y que siempre se deben intentar recordar en todo trabajo científico. Hay que tener presente que los fundamentos de las conclusiones de nuestras investigaciones provienen, en gran medida, de los datos que se analizan, si éstos son defectuosos, nuestras valoraciones también lo serán. Como afirman los profesores CASAS y FLORISTAN (1953): "lo primero que se le exige a 1 investigador es honradez profesional". Esta frase hay que tenerla muy en cuenta en todos los trabajos que se realicen, tanto para citar la fuente de la que proviene la idea como para comprobar su fiabilidad.

Lógicamente si con las informaciones que se está trabajando son de una escala local siempre se pueden cotejar con la realidad más fácilmente que si nos movemos a otras escalas de información, más difíciles de comprobar, aunque queda la posibilidad de comparar con otra fuente.

La intención de este artículo es dar a conocer algunos de los problemas con los que se pueden encontrar otros investigadores que acudan a la consulta de las mismas fuentes que aquí mencionamos, a los cuales estas reflexiones les pueden servir de apoyo en la forma de actuar en su manejo.

\section{INCONVENIENTES GENERALES DE LAS FUENTES ESTADÍSTICAS}

Un primer aspecto a destacar es una serie de problemas generales con los que se tiene que enfrentar el investigador en relación con el tema de las estadísticas y que no son exclusivos de los trabajos sobre el análisis de la localización industrial:

- Las diferencias en las informaciones para un misno dato. Por ejemplo, si estamos buscando el número de obreros de una determinada empresa y consultamos dos fuentes distintas, la información puede no coincidir, bien por una diferencia en las fechas de contabilización de esas cifras, bien por un error a la hora de transcribir las informaciones desde cada empresa, o simplemente por el empleo de distintos métodos a la hora de contabilizar ese dato. 
Este primer problema exige ya una ponderación de la fiabilidad de cada fuente, aceptando las cifras de aquella que ofrezca una mayor credibilidad. En nuestros trabajos deberemos anotar siempre las discrepancias e incoherencias que se vayan detectando en las fuentes consultadas, tanto para ayudar a conocerla mejor a otros investigadores, como para que en nuestras conclusiones quede constancia de ello.

- Otro inconveniente grave con el que nos podemos encontrar es con la inexistencia de los datos que se precisan para completar nuestros objetivos, lo cual obliga a limitar el trabajo a lo que las fuentes ofrecen. Por ello, como ya se ha comentado, no viene nada mal antes de abordar el esquema de un trabajo plantear la información con la que se puede contar.

- La inaccesibilidad para conseguir informaciones. En ocasiones, por distintos motivos, la Administración no permite consultar determinados datos, como es el caso de los listados empresariales del Ministerio de Hacienda, cuyos inconvenientes se verán más adelante; también muchos organismos privados son reacios a dar informaciones sobres sus actividades, aún garantizando que éstas sólo se van a utilizar para una labor de investigación.

- La falta de organización y sistematización de una nisma fuente es otro problema al que se enfrenta el investigador. Esto le exige conocerla previamente para obtener lo que requiere y extraerlo con facilidad. En esta sistematización hay que examinar 1 a seriación, la periodicidad y homogeneidad que entre unos y otros años puede presentar una misma fuente, sobre todo si el seguimiento que se va a realizar es evolutivo.

- Hay que considerar también la escala de trabajo y consultar que fuente se puede conseguir para esa escala, ya que dependiendo de ésta los datos que aportan pueden ser diferentes.

- Los errores a la hora de recopilar las informaciones. Hoy cada vez son más las bases de datos que se pueden encontrar informatizadas, con lo cual va disminuyendo 1 a posibilidad de que se cometan errores a la hora de recopilar información, aunque ello no disminuye los fallos que muchas veces incorporan las bases de datos informatizadas, y que pueden aportar importantes aberraciones a nuestros trabajos. Siempre es conveniente examinar con cierta tranquilidad tanto las informaciones que se tomen de forma manual como las que provengan de distintas bases informatizadas, valorando que cada dato recopilado es esencial para nuestros resultados.

Todos estos aspectos deben ser tenidos en cuenta de forma general en nuestras investigaciones, pero de esto tampoco se debe deducir que si no tenemos información demasiado fiable sobre algún aspecto no se debe utilizar. Cuando esto ocurre, lo que se indicará son todas aquellas anomalías, errores y demás aspectos destacables que en su 
análisis se hayan detectado, y si no existe otra estadística emplear la que se dispone, pero con cierta prudencia en las conclusiones que se obtengan de ella, y por supuesto acudir a la encuesta y al trabajo de campo para las comprobaciones.

\section{FUENTES PRINCIPALES PARA EL ESTUDIO DE LA LOCALIZACIÓN INDUSTRIAL}

En las investigaciones sobre temas de localización industrial se puede trabajar desde datos a nivel continental hasta la escala local, siendo estos últimos los más difíciles de recopilar y de conseguir, ya que suelen ser datos más particulares y por tanto más complicados de obtener, pues casi siempre se tiene que acudir a las fuentes directas frara conseguirlos. Sin embargo, la escala municipal tiene la ventaja de obligar al investigador al contacto directo con el territorio, lo que le permite conocer mejor su realidad, comprobando todo aquello que los documentos no reflejan, en definitiva, permitiendo un análisis más valioso. En este articulo nos centramos principalmente en la revisión de las fuentes que en esta escala se pueden consultar, para ello se ha utilizado como territorio de estudio la región aragonesa, pero la situación no varia mucho a la de otras regiones españolas.

El análisis de las fuentes sobre localización industrial ha sido un tema muy pocas veces abordado desde la perspectiva geográfica $y$, sin embargo, es sin duda una de las mayores dificultades que se plantean en los estudios geoindustriales. Tal es la importancia de su escasez en nuestro pais, que incluso se ha considerado que es una de las principales causas del escaso interés prestado al análisis del sector industrial por parte de los geógrafos españoles (CARAVACA, 1987).

Durante los años ochenta y noventa, las investigaciones sobre Geografía Industrial se han ido incrementando en nuestro país, aunque no por el aumento de estadísticas para su estudio, sino porque se ha ido viendo la importante relación entre la transformación del territorio y esta actividad. A pesar de la escasez, errores y de la dificultad que supone conseguir información desagregada territorialmente sobre el sector industrial, la imperiosa necesidad de su análisis obliga a trabajar con las fuentes que existen.

Cuando revisamos las estadísticas publicadas sobre el sector industrial en escalas espaciales amplias o tomando niveles de empresas de muchos empleados ha y bastante información, así por ejemplo existen publicaciones periódicas de prácticamente todas las ramas industriales, realizadas bien por la correspondiente Secretaria General del Ministerio de Industria, bien por distintos organismos privados a través de anuarios. Estas fuentes a veces nos pueden ofrecer informaciones puntuales 
sobre una empresa con importancia a nivel nacional, bien por su volumen de facturación o número de empleos, pero en la mayoría de ocasiones no nos sirven para acercamos a cuestiones de localización (HERRERO y PALLARDO, 1988).

En escalas espaciales más próximas al territorio no existe ninguna publicación periódica con estadísticas sobre este sector, lo que obliga a establecer contactos directos con los propios establecimientos empresariales o con los organismos públicos que poseen la información más desagregada.

Si son datos que no se encuentran recogidos en listados por ninguna Administración ni publicados, se tienen que obtener a través de la investigación directa, tarea difícil para el investigador, ya que las empresas guardan muy en secreto ciertas informaciones que ellas consideran como confidenciales, por ejemplo, su organización, sus distribuidores, sus clientes, sus filiales, la procedencia de su mano de obra, etc...

Para obtener estos datos se puede optar por dos alternativas; la primera, en el caso de que sean bastantes las empresas que tenemos que analizar, realizar una encuesta a todas ellas y esperar a que te contesten si es que se ha mandado por correo, 0 esperar a que te reciban si es que se realiza de forma directa, esta última suele ser más positiva porque siempre se pueden conseguir informaciones adicionales al realizarla. La segunda, en el caso de que sólo se trate de una o varias empresas, lo mejor es dirigirse a ellas, intentando conseguir que nos reciba el gerente, y entonces el que nos faciliten la información que se solicita depende de la persona que esté al cargo de la empresa.

En cuanto a las informaciones que se encuentran recogidas en los distintos organismos públicos (Ministerios, Cámaras de Comercio e Industria, Ayuntamientos, ...) dependiendo de cada caso, tienen más o menos accesibilidad. A continuación planteamos las dificultades principales con las que nos podemos encontrar en la consulta de estas fuentes para el correcto estudio del sector industrial en Aragón y que, como ya se ha comentado, pueden servir para ser tenidas en cuenta en otros territorios.

\section{a) Ministerio de Hacienda y Tesorería de la Seguridad Social}

Lo primero que hay que señalar sobre las bases de datos del Ministerio de Hacienda es que tienen carácter fiscal, por lo que no son accesibles para ningún tipo de investigación, ya que están bajo la Ley de Protección de Datos Informáticos, que prohibe dar información a cualquier entidad que no tenga fines recaudatorios. Se cierra por tanto la posibilidad de consulta de esta fuente, que hubiera sido quizás la más próxima a la realidad, por su fiabilidad económica, puesto que toda empresa tiene obligación de figurar en las licencias fiscales de Hacienda. 
Otra fuente que posee información útil para el análisis del sector industrial es la Tesorería de la Seguridad Social, sus bases de datos tienen también carácter fiscal, pues toda empresa debe tener al día su situación laboral. Este organismo está acogido actualmente a la Ley de Protección de Datos Informáticos, por lo que de él se obtiene respuesta negativa para la consulta de sus bases de información. Esta fuente ha sido muy usada durante años para acceder a información sobre el empleo industrial a través de las mutualidades laborales, sin embargo, en la actualidad se acogen a esa Ley y se niegan a facilitarla.

Ninguna de estas dos bases de datos anteriores se pueden conseguir en estos momentos, por lo que no se insiste más en ellas, ante la imposibilidad de utilizarlas ni siquiera para la realización de trabajos académicos.

\section{b) Cámaras de Comercio e Industria}

Las Cámaras de Comercio e Industria, desde hace unos años, tramitan una serie de pagos de las distintas empresas, por lo que deben conseguir una base de datos lo más fiable posible, y acuden para conseguirlas tanto a los Gobiernos de las Comunidades Autónomas como al Ministerio de Hacienda.

Uno de los inconvenientes que presenta este fuente es que cada Cámara de Comercio e Industria posee una circunscripción territorial, por lo que si se necesitan informaciones que correspondan a varias Cámaras, el conseguir éstas se complica, sobre todo por la diferente estructuración interna de estos organismos. El acceso es variable, depende de cada caso, además también hay que hacer las consiguientes peticiones por escrito para acceder a ellas, por lo que en cada caso habrá que estudiar previamente como funciona. Para el caso de Aragón, cada provincia tiene su propia Cámara de Comercio. En la de la provincia de Zaragoza se pueden obtener en soporte informático listados que contienen distintos epígrafes con informaciones concretas sobre cada empresa: razón social, dirección, código postal, municipio, IAE (Impuesto de Actividades Económicas), descripción de la actividad que desarrolla y número de obreros.

Esta misma fuente y con los mismos epígrafes, ofrece también información sobre el sector servicios, útil para analizar algunas de las transformaciones que en este sector se han producido en relación con las implantaciones industriales.

Las informaciones que aportan las Cámaras de Comercio podían ser más útiles, ya que con ellas no se pueden establecer comparaciones ni evoluciones con años anteriores puesto que sólo conservan los últimos datos informatizados, ni tampoco se trata de una base publicada por lo que no tiene periodicidad. Por tanto, esta fuente se puede utilizar para aquellos datos de los que sólo se busca ofrecer una referencia de un 
momento concreto (por ejemplo, la localización actual de la industria en un municipio o en la provincia).

c) Gobiernos Autonómicos

El Gobierno de la Comunidad Autónoma de Aragón, a través de los servicios de promoción industrial, también posee un registro sobre las empresas que existen en la comunidad. Aunque la finalidad de este registro es simplemente de carácter informativo posee datos sobre todas las empresas de la Comunidad Autónoma. El acceso a esta información varía según la persona que esté al frente de estos servicios, que puede facilitarnos los datos de los que disponen o bien pueden negarlos diciendo que es para uso interno de la Administración.

En el caso del Servicio de Promoción Industrial de la Diputación General de Aragón, tras varios viajes y entreviștas se consiguió, previo pago de una cantidad por empresa, acceder a una muestra de varios municipios del sistema de información empresarial del que disponen. ésta se utilizó para comparar con las otras bases de datos de las que se disponía. Los epígrafes de esta fuente son: CNAE (Código Nacional de Actividades Económicas), razón social, nombre del gerente, dirección, población, código postal, provincia, teléfono, número de trabajadores y productos que fabrica cada empresa.

Estos epígrafes son prácticamente los mismos que los que posee la Cámara de Comercio e Industria, con la que no se tiene ningún problema de acceso, además de proporcionarlos gratis. A esto hay que añadir que por las comprobaciones que entre una y otra se hicieron, es más completa la de la Cámara que la del Servicio de Promoción Industrial, fuente en la que se le encontraron algunos errores, tanto de empresas que hacia años que habían dejado de funcionar y todavía figuraban en sus listados, como la falta de algunas empresas existentes. Sin embargo, se necesita consultar esta fuente para aquellos casos en los que el análisis es de todo Aragón.

Para cada Comunidad Autónoma en 1991 los servicios de promoción industrial junto con el IMPI (Instituto de la Mediana y Pequeña Industria) publicaron un Directorio Industrial. En él están incluidos los siguientes epígrafes: nombre de la empresa, dirección, población, teléfono, gerente, ventas, actividad, materiales que emplea y productos que fabrica.

La ventaja de esta fuente es que al estar publicada su acceso es más fácil y rápido para una consulta concreta, ya que contiene varios índices: índice alfabético por empresas, índice alfabético de empresas por provincia y municipio, índice de especialidades e índicu de productos. Sin embargo, este Directorio no se facilita informatizado, por lo que trabajar con é! puede recultar más costoso. De cada municipio 
se posee la relación de empresas en el índice, ordenadas a través del número de ficha de cada empresa, aunque en el texto hay que buscar en distintos apartados estas empresas, ya que esta fuente está ordenada por sectores de actividad y no por municipios, lo cual dificulta en algunas ocasiones su utilización. No cuenta con antecedentes, por lo que no sirve para ver evoluciones, pero sí para consulta de datos concretos sobre las empresas. Su estructura es igual para todas las Comunidades Autónomas, en ella sólo aparecen las empresas con más de 5 empleados, hecho que limita su utilización en una Comunidad Autónoma como Aragón, donde predomina la pequeña empresa. Contiene bastantes ausencias, aunque incorpora un epígrafe que hasta ahora no nos había aparecido en ninguna fuente como es el volumen de ventas, dato, que pese a los posibles errores que pueda contener, nos da una aproximación bastante buena de la importancia de la empresa.

Otro inconveniente es que de cada empresa no se aporta ni el CNAE ni el IAE, por lo que sólo podemos, en principio, plantear con ellas una clasificación sectorial amplia. Esta fuente es, sobre todo, un documento de consulta sobre alguna información concreta de los establecimientos que contiene.

A las bases de datos anteriores hay que añadirle otra que los gobiernos autonómicos, dentro de sus departamentos de agricultura, ganadería y montes, durante muchos años han venido realizando, el Directorio de Empresas Agroalimentarias que desde el año 1994 se intenta homogeneizar con los datos del Registro Industrial.

La información que contiene la base de datos de este Departamento se caracteriza por la fiabilidad de sus contenidos, puesto que se ha ido elaborando con un importante control por parte de la Administración. En ella se incluyen los siguientes epígrafes: localidad, código de la comarca a la que pertenece, domicilio social, clase de actividad (cárnica, etnológica, hortofrutícola, etc...), titular, actividades (hasta nueve diferentes), puestos de trabajo fijos y jornales eventuales. En relación con el último epígrafe, hay que señalar la importancia que en las industrias agroalimentarias tiene el empleo eventual, ya que algunas de las actividades que desarrollan se realizan en épocas concretas del año, que es cuando se precisan mayor número de trabajadores, por lo que el conocimiento de este dato puede resultar muy interesante si se analiza con más profundidad este sector.

d) Ministerio de Industria y Energía

El Ministerio de Industria y Energía (MINER) cuenta con la base de datos más utilizada para los análisis industriales, el Registro Industrial. Esta fuente es in registro administrativo, en él se incluyen los establecimientos industriales de todas las provincias españolas. Teóricamente esta fuente tenía que permitir efectuar un seguimiento detallado del sector industrial, puesto que se viene realizando desde hace 
bastantes años y aporta informaciones que sólo ella contiene. El Registro Industrial se ubica en Madrid dentro del Ministerio de Industria en su Secretaría General Técnica, allí, se encuentran centralizados y compendiados todos los registros provinciales. Es por tanto, una de las pocas fuentes industriales detalladas para todo el ámbito nacional (MOMPO y MONFORT, 1989).

Los sectores industriales incluidos en él son prácticamente todos, salvo la industria de producción y distribución de energía eléctrica y la minería que siempre a la hora de realizar su inscripción plantean problemas por sus características especiales. Tampoco se encuentran en él muchas industrias agropecuarias y forestales, que dependen del Ministerio de Agricultura, Pesca y Alimentación. Cada una de estas actividades está incluida en registros especiales del Ministerio de Industria y del Ministerio de Agricultura respectivamente (JARNER y PICADO, 1982). Del mismo modo, en el Registro Industrial se centraliza la información de algún otro sector que no es actividad industrial propiamente dicha, como es el caso de los talleres de reparación o las empresas de transporte. La inclusión de estas actividades no plantea problemas al venir definidas también por el CNAE, así si no interesa tenerlas en cuenta se pueden suprimir.

La fiabilidad de esta fuente se aprecia de forma más clara si se examina su forma de funcionar. La legislación española obliga a toda empresa que se cree, se amplíe o se modifique, a inscribirse en este Registro (Real Decreto 2135/80 de 26 de septiembre de $19^{\circ} 0$ ). Los datos que proceden de esta fuente son rellenados por un administrativo con la información que facilita cada empresa, por lo que en gran parte su grado de utilidad dependerá de la declaración que ésta realice. Este punto es el primero que hace falta anotar para utilizar esta fuente con cautela, ya que aunque la Administración inspecciona las instalaciones, hay veces que después de pasada la inspección algunos establecimientos modifican sus características iniciales.

Los formularios de todas las empresas inscritas en el Registro Industrial son explotadas por el MINER desde 1939, pero es en 1965 cuando se inicio la informatización de la inscripciones. En principio, se empezaron a recopilar en archivos con ficha perforada, donde cada empresa tenía sus datos sobre los movimientos que iba realizando. Estos ficheros se conservan en algunas Direcciones Provinciales del MINER, e incluyen las fichas de empresas que hace muchos años que desaparecieron. $\mathrm{Si}$ estos ficheros estuvieran actualizados sería la mejor fuente para hacer el seguimiento de una empresa, pero su falta de puesta al día impide la generalización de su uso. Al comenzarse la informatización de esta fuente, se empezó a obtener una renovación continua de la base de datos, borrando empresas que se dan de baja y anotando nuevas empresas, por lo que no se puede hacer un seguimiento. 
Sus datos se explotan de varias formas: primeramente, a través del también denominado Registro Industrial que contiene los siguientes epígrafes: número de inscripción en el registro, CNAE, razón social, emplazamiento, código postal, municipio, año de instalación o último movimiento registrado, potencia instalada (kw) y número de trabajadores. Todos estos datos resultan muy útiles para dar una visión del sector industrial y su distribución en cualquier escala espacial (municipal, provincial, regional o nacional). Esta forma de explotación recoge todos los expedientes de las industrias inscritas, y con ellos se preparan unos listados provinciales ordenados según los distintos epígrafes: por el número de inscripción en e 1 Registro, por orden alfabético de las empresas, por las actividades según el CNAE y por municipios. Estos se pueden encontrar en las Direcciones Provinciales o en el MINER en Madrid.

La segunda forma de explotación de los datos del Registro Industrial es el Moviniento Industrial anual, extraído a través de las fichas de nuevas industrias y de las ampliaciones realizadas en un determinado periodo. Esta información se puede consultar trimestralmente a través de la Revista Economía Industrial, que recoge las informaciones de estos movimientos o bien, si es para un estudio concreto, se puede solicitar dicha información directamente a la sección del Registro Industrial, aunque actualmente se encuentran en una etapa de cambios y no la facilita. Los epígrafes que incluye son: tipo de expediente ( 2 = nueva industria, 4 = ampliación, $6=$ revisión, b= baja), código de la provincia, número de inscripción en el registro, fecha, CNAE, razón social, dirección, municipio, código postal, potencia total, número de empleados e inversiones en capital fijo (terrenos, maquinaria, ...).

A través del Movimiento Industrial se pueden obtener unas informaciones muy valiosas, como son: las empresas de nueva creación cada año, su potencia, el número de obreros, su actividad, etc... y todo ello se extrae por el tipo de expediente de cada empresa, tomándose en este caso el tipo 2, es decir, nuevas empresas.

A estas dos explotaciones informáticas hay que añadir las Tablas de Frecuencia que es una presentación de las distintas actividades por provincias según su personal y potencia instalada. Estas se llevan a cabc mensual y trimestralmente y son publicadas también en la Revista Economía Industrial del MINER.

El Registro Industrial se convierte en una fuente muy importante a la hora de estudiar el sector industrial, porque además de aportar datos que las otras fuentes no ofrecen (inversión, potencia o el movimiento industrial anual), está constantemente configurándose. Tal y como está planteado se puede analizar anualmente el movimiento industrial (altas y bajas), o se pueden consultar las explotaciones periódicas que existen. 
Sin embargo, esta fuente para su correcta utilización precisa plantear previamente toda una serie de matizaciones, puesto que contiene bastantes inconvenientes para su uso tal y como plantean MOMPO y MONFORT en un artículo sobre el Registro Industrial en la Comunidad Valenciana (MOMPO y MONFORT, 1989), aplicables al caso a aragonés, y que anotamos brevemente:

- En el Registro Industrial no están contabilizadas muchas de las actividades mineras, cuya inscripción se realiza en un registro propio (Registro Minero) con rasgos similares al Registro Industrial, donde además aparecen incluidas algunas otras actividades propiamente industriales como algunas siderúrgicas, fábricas de cementos, fabricación de materiales de construcción en cemento, yeso o escayola, empresas de obras públicas etc... que desde hace tiempo se inscribieron en el Registro Minero, por utilizar minerales en su producción, y hoy por rutina todavía continúan inscritas en él y no en el Registro Industrial. Esto provoca que ninguno de ambos registros aporten la información total de los sectores que teóricamente tenían que incluir. La solución para evitar las duplicidades que se producen entre ambos registros (minero e industrial) sería la de unificarlos, ya que se podrían extraer las informaciones que interesen a través del CNAE.

- En las actividades agrarias y alimentarias, debido a que el Ministerio de Agricultura lleva su propia contabilización de establecimientos agroalimentarios, el Registro Industrial contiene bastantes deficiencias en las inscripciones de este tipo de industrias, así algunas empresas de este sector si que aparecen pero otras no. Durante años, este tipo de industrias estuvo bastante descuidado por parte del Registro Industrial, aunque desde hace unos pocos años el registro de todas estas empresas también se realiza en el Registro Industrial, existiendo aun con todo algunos defectos en las inscripciones de este sector.

- Las empresas de producción y distribución de energía eléctrica también plantean problemas, no tiene una buena comunicación con el Registro Industrial, ya que tienen una sección especial dentro de la Administración para tramitar todos los asuntos relacionados con ellas por ser actividades con una legislación especial. Sin embargo, esta sección si que mantiene una buena base de datos sobre el sector, con lo cual si se precisa información sobre él es mejor acudir a ella directamente.

- Pero, quizás, el defecto más grave que contiene el Registro Industrial y que afecta a todos los sectores es el relacionado con el control de bajas, puesto que el procedimiento administrativo actual es muy poco exigente para que las empresas acudan a comunicar su cese de actividad. A pesar de existir la obligación de inscribirse en el Registro Industrial para la apertura de la empresa y también de comunicar su baja cuando cierra, muchas veces no se realiza esto último. Por ello, en esta fuente pueden aparecer un número muy elevado de empresas que hace años que ya no existen y 
que al no tener ninguna presión para darse de baja, siguen sin hacerlo. La única solución es que la Administración pusiera algún tipo de obligación a las empresas para que se dieran de baja.

- Otro de los inconvenientes que tiene el Registro Industrial esta relacionado con la inscripción de distintos establecimientos que pertenecen a una misma empresa, figurando en el registro tantas veces como establecimientos tenga la empresa, y puede incluso figurar en tantas ocasiones como actividades diferentes desarrolle. Esto se soluciona ordenando por direcciones o por nombre de la empresa la base de datos.

- En relación con el apartado de inversiones, hay que tener presente que se pueden producir ocultaciones por parte de las empresas, ya que después de pasada 1 a inspección éstas pueden aumentarlas. El incremento de inversión de una empresa ya creada, que entraría dentro del apartado de Ampliaciones, no suele ser registrado, bien por desconocimiento, dejadez o por no querer hacerlo. Así, la mayoría de ampliaciones que se registran es porque las empresas han aumentado su potencia y para esto la compañía eléctrica les exige tener tramitada la correspondiente autorización de la Administración. Esto es así porque no existe una normativa que controle y sancione todas estas infracciones.

Tanto el Registro Industrial como el Movimiento Industrial podían ser las bases de datos más valiosas sobre el sector industrial, sin embargo, todas las limitaciones que ya se han planteado sobre ellas invalidan bastante los resultados que se podían obtener, aunque esto no impide su utilización, siempre y cuando se tengan siempre en cuenta sus inconvenientes, intentando poner solución a algunas cuestiones, tanto con trabajo de campo como a través de la comprobación de aquellas empresas que llevan muchos años en funcionamiento. Hay que tener en cuenta que a pesar de sus errores, hay informaciones que sólo se pueden conseguir de estas fuentes (inversiones, evolución de nuevas empresas, etc...) y que son muy precisas para el estudio de las localizaciones industriales, por lo que no queda otro remedio que trabajar con sus datos, que se tomarán siempre con mucha cautela, y sólo como indicadores de la situación real. Estas fuentes son además muy válidas para el estudio desagregado de la actividad industrial a distintas escalas territoriales, a través de ellas podemos partir desde el análisis municipal hasta el nacional, con una información muy rica desglosada por sectores a través del CNAE.

\section{VALORACIÓN SOBRE LAS FUENTES DEL SECTOR INDUSTRIAL}

Como se ha comprobado el panorama de las fuentes que se pueden consultar para llevar a cabo estudios sobre la actividad industrial no es demasiado favorable, ya que 
hay más aspectos negativos que positivos. Pero éstos no deben detener nucstra investigación, ni desanimamos, sino que una vez conocidos los posibles errores, y si éstos no se pueden solucionar por el investigador, utilizaremos la fuente de la que dispongamos, aunque siempre tendremos cautela con las interpretaciones extraídas de esas informaciones.

Junto a todas las indicaciones ya apuntadas en cada una las fuentes observadas en este artículo, cabe plantear algunas otras generales que completan la lista de inconvenientes que presentan todavía hoy las fuentes para Geografía Industrial y que pueden ser muy útiles para quienes se acerque al estudio de estos temas.

- La dificultad de elegir la fuente a utilizar; aunque son todavía escasas las estadísticas sobre el sector industrial, pueden existir varias para un mismo dato. Ello plantea la necesidad de elegir en cada momento aquella que sea más fiable, más actualizada, o la que contenga el dato que se busca. En función de las necesidades se empleará una u otra, pero en todas hay que tener en cuenta su grado de fiabilidad, y hacer siempre alusión a su procedencia.

- La imposibilidad de comparación entre fuentes; como se observa repasando los ejemplos examinados, tanto la disparidad de criterios con los que están elaboradas cada fuente como la no coincidencia de epígrafes, hace muy difícil su comparación.

- El difícil acceso a las distintas estadísticas, puesto que son bases de datos que cada uno de los organismos posee para su uso interno. Salvo en el caso del Directorio Industrial que es una fuente publicada, para el resto de los ejemplos observados son necesarios largos procesos burocráticos con las consiguientes peticiones y declaraciones de que el uso de las informaciones solicitadas son para la realización de un trabajo académico.

- El desconocimiento "a priori" de las fuentes existentes, no sólo en cuanto a su grado de fiabilidad sino en la forma de poder conseguirlas. Sería conveniente conocer previamente la fuente que se va a consultar, averiguar si los datos que precisamos se pueden conseguir a través de las redes informáticas o localizar las distintas bases de datos informatizadas de los distintos organismos. Para ello, lógicamente, hace falta saber que existe esa información, lo cual a veces no es tan fácil, porque los propios encargados de los organismos desconocen a veces la información que su servicio posee.

- La dispersión de las distintas informaciones. Al no existir centralización de 1 a información los distintos organismos se plantean sus propias bases de datos. 
- La falta de desagregación territorial de muchas informaciones, lo cual limita mucho los distintos trabajos que se quieran plantear, así como el número de fuentes de consulta.

- La imposibilidad de ofrecer información actualizada, ya que es constante el movimiento de altas y bajas, ampliaciones y renovaciones que se producen el mundo empresarial diariamente. Muchas veces se tiene que trabajar con los datos desfasados.

- La desconfianza por parte de los distintos organismos y empresas privadas a facilitar información. Esto provoca que no se puedan conseguir datos que nos hubieran ayudado mucho en nuestro trabajo. Las excusas siempre van en relación con la confidencialidad de las informaciones.

- La falta de continuidad de algunas publicaciones, esto impide tener posibilidad por un lado de establecer una comparación con datos anteriores y por otro de obtener una valoración crítica de esa fuente.

- La falta de una definición de lo que son actividades industriales, lo que provoca la inclusión de distintas actividades que no pertenecen al sector. En relación con esto hay que hacer referencia a las transformaciones acaecidas en el actual sistema productivo que conlleva una fuerte interrelación de las actividades productivas, lo que genera muchas dudas sobre qué actividades incluir en el epígrafe de industriales.

\section{REFLEXIONES FINALES}

Es patente que, a pesar de la evolución acometida en los aspectos relacionados con las bases de datos gracias a los medios informáticos, son todavía muchos los aspectos relacionados con las fuentes estadísticas que siguen haciéndose plantear la necesidad del control de su fiabilidad. Por ello, todas las informaciones tomadas de las distintas fuentes deben incorporar su procedencia, para que la persona que las examine pueda valorar su fiabilidad, norma importante a seguir en todo trabajo de investigación.

En definitiva, si difícil es plantearse un trabajo de investigación, más lo es cuando se tienen que utilizar fuentes que son inaccesibles, o contienen bastantes errores, como es el caso de las fuentes existentes para el sector industrial. Todo ello no facilita la labor de ir hacia adelante, al contrario, muchas veces la falta de información o el retraso en conseguirla dificulta enormemente. Esto origina que cuando hay que afrontar una situación de búsqueda de datos se tenga que emplear mucho tiempo, puesto que no sólo hay que saber la información que se necesita sino saber si existe y donde está. 
Es evidente la importancia que tiene la información en cualquier tipo de investigación, sin embargo, en ella el investigador sólo puede actuar como un consumidor de una información, y que sólo a través del trabajo de campo con comprobaciones puede modificarse, indicando que la fuente original ha sufrido modificaciones.

Todos los aspectos que se han comentado sobre las fuentes de información para el análisis del sector industrial ponen de relieve la importancia que tiene un buen conocimiento y soltura en el manejo de las mismas. Se puede afirmar que quien tiene estos requisitos tienen casi garantizado un buen trabajo o por lo menos un trabajo fácil.

\section{BIBLIOGRAFÍA}

ALONSO LOGROÑ , M". P. (1996): Procesos de difusión axial: la industria en Zaragoza. Prensas Universitarias. Universidad de Zaragoza. Microfichas, serie Tesis doctoral. $1098 \mathrm{p}$.

CARAVACA BARROSO, I. (1987): "La investigación en Geografía Industrial y su evolución en España". Finisterre, vol. XII, 44, 385-397. Lisboa.

CASAS, J.M. y FLORISTAN, A. (1953): "Iniciación a la Geografía local". C.S.I.C., Departamento de Geografía Aplicada, 166 p, Zaragoza.

HERRERO, R. y PALLARDO, F. (1988): "Aproximación a las fuentes de información de la economía industrial española". Rev. Economía Industrial, marzo-abril, 123-135, Ministerio de Industria y Energía, Madrid.

JARNER, J. M. y PICADO, M. E. (1982): "El registro industrial". Rev. Economía Industrial, Diciembre 1982, 66-71. Ministerio de Industria y Energía.

MOMPO, A. y MONFORT, V. (1989): "El Registro Industrial como fuente estadística regional: el caso de la Comunidad Valenciana". Reo. Economía Industrial, 268, 129-137. Ministerio de Industria y Energía.

RÍO, I. del (1986): "Notas sobre la evolución del contenido y los métodos de la Geografía Industrial". En GARCIA BALLESTEROS, A.: Teoría y práctica de la Geografía. Ed. Alhambra, 284- 292. Madrid.

RUIZ ARBE, A. (1995): "La información estadística el la economía aragonesa". III Congreso de Economía Aragonesa. Facultad de Económicas y Empresariales y Gobierno de Aragón, 10 p. Zaragoza. 